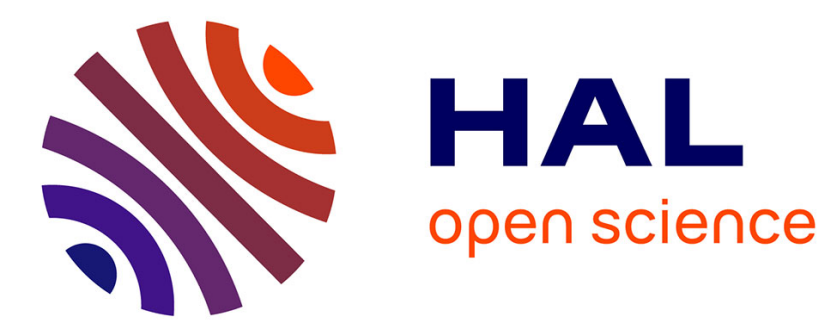

\title{
Towards the molecular understanding of glycogen elongation by amylosucrase.
}

Cécile Albenne, Lars K Skov, Vinh Tran, Michael Gajhede, Pierre Monsan, Magali Remaud Simeon, Gwenaëlle André-Leroux

\section{To cite this version:}

Cécile Albenne, Lars K Skov, Vinh Tran, Michael Gajhede, Pierre Monsan, et al.. Towards the molecular understanding of glycogen elongation by amylosucrase.. Proteins - Structure, Function and Bioinformatics, 2007, 66 (1), pp.118-26. 10.1002/prot.21083 . hal-02663870

\section{HAL Id: hal-02663870 \\ https://hal.inrae.fr/hal-02663870}

Submitted on 31 May 2020

HAL is a multi-disciplinary open access archive for the deposit and dissemination of scientific research documents, whether they are published or not. The documents may come from teaching and research institutions in France or abroad, or from public or private research centers.
L'archive ouverte pluridisciplinaire HAL, est destinée au dépôt et à la diffusion de documents scientifiques de niveau recherche, publiés ou non, émanant des établissements d'enseignement et de recherche français ou étrangers, des laboratoires publics ou privés.

\section{다(1)(2)}

Distributed under a Creative Commons Attribution - ShareAlike| 4.0 International 


\title{
Towards the Molecular Understanding of Glycogen Elongation by Amylosucrase
}

\author{
Cécile Albenne, ${ }^{1}$ Lars K. Skov, ${ }^{2}$ Vinh Tran, ${ }^{3}$ Michael Gajhede, ${ }^{2}$ Pierre Monsan, ${ }^{4}$ Magali Remaud-Siméon, ${ }^{4 *}$ \\ and Gwénaëlle André-Leroux ${ }^{5}$ \\ ${ }^{1}$ Laboratoire Surfaces Cellulaires et Signalisation chez les Végétaux, UMR 5546 CNRS-Université Paul Sabatier-Toulouse III, \\ 24 Chemin de Borde Rouge, BP42617, 31326 Castanet-Tolosan, France \\ ${ }^{2}$ Biostructural Research, Department of Medicinal Chemistry, The Danish University of Pharmaceutical Sciences, \\ Universitetsparken2, DK-2100 Copenhagen, Denmark \\ ${ }^{3}$ Unité Biotechnologie, Biocatalyse, Biorégulation (U3B) UMR-CNRS 6204, Université de Sciences et Techniques de Nantes, \\ 2, rue de la Houssinière, 44322 Nantes, France \\ ${ }^{4}$ Laboratoire de Biotechnologie-Bioprocédés, UMR CNRS 5504, UMR INRA 792, INSA DGBA, 135, avenue de Rangueil, \\ 31077 Toulouse Cedex 04, France \\ ${ }^{5}$ INRA, Institut Pasteur, Unité de Biochimie Structurale, 25, rue du Dr Roux, 75724 Paris Cedex 15, France
}

ABSTRACT Amylosucrase from Neisseria polysaccharea (AS) is a transglucosidase from the glycoside-hydrolase family 13 that catalyzes the synthesis of an amylose-like polymer from sucrose, without any primer. Its affinity towards glycogen is particularly noteworthy since glycogen is the best D-glucosyl unit acceptor and the most efficient activator (98-fold $k_{\text {cat }}$ increase) known for this enzyme. Glycogen-enzyme interactions were modeled starting from the crystallographic AS: maltoheptaose complex, where two key oligosaccharide binding sites, $\mathrm{OB} 1$ and $\mathrm{OB} 2$, were identified. Two maltoheptaose molecules were connected by an $\alpha-1,6$ branch by molecular modeling to mimic a glycogen branching. Among the various docking positions obtained, four models were chosen based on geometry and energy criteria. Robotics calculations enabled us to describe a back and forth motion of a hairpin loop of the AS specific $B^{\prime}$-domain, a movement that assists the elongation of glycogen branches. Modeling data combined with site-directed mutagenesis experiments revealed that the $\mathrm{OB} 2$ surface site provides an anchoring platform at the enzyme surface to capture the polymer and direct the branches towards the OB1 acceptor site for elongation. On the basis of the data obtained, a semiprocessive glycogen elongation mechanism can be proposed. Proteins 2007;66:118-126. $\odot 2006$ Wiley-Liss, Inc.

Key words: molecular modeling; robotics; docking; transglucosylation; site-directed mutagenesis; enzymatic mechanism

\section{INTRODUCTION}

Amylosucrase (E.C. 2.4.1.4) from Neisseria polysaccharea (AS) is a bacterial transglucosidase from glycoside hydrolase family 13 , also named the $\alpha$-amylase family. ${ }^{1-3} \mathrm{AS}$ is closely related to other transferases of this family such as CGTase or amylomaltase; however, it is the only polymerase. AS catalyzes the synthesis of an insoluble amylose-like polymer from sucrose with concomitant release of fructose. ${ }^{1}$ This reaction starts by sucrose hydrolysis. The glucose formed is in turn used as an acceptor that is then successively elongated to produce a series of maltooligosaccharides that are soluble until their size and concentration cause their precipitation. ${ }^{4,5}$ In contrast, when glycogen is added into the medium at a concentration higher than $0.5 \mathrm{~g} / \mathrm{L}$, neither glucose nor oligosaccharide formation is observed. ${ }^{6}$ The glucosyl units of sucrose are transferred exclusively onto some glycogen branches until a critical size causing polymer insolubilization. For example, the modified glycogen formed from $146 \mathrm{~m} M$ sucrose and $1 \mathrm{~g} / \mathrm{L}$ glycogen displays some branches with a degree of polymerization (DP) of $75 .{ }^{6}$ Moreover, a strong increase of the sucrose consumption rate is observed in the presence of glycogen. ${ }^{6}$ This activation is dependant on both sucrose and glycogen concentrations. For example, using $106 \mathrm{mM}$ sucrose, and $30 \mathrm{~g} / \mathrm{L}$ glycogen, a 98 -fold increase of sucrose consumption rate is obtained. ${ }^{6}$ Other $\alpha-1,4$ glucans can also be modified via glucosylation by $\mathrm{AS},{ }^{7}$ but they were shown, so far, to be less efficient activators. This strongly suggests that the in vivo function of AS could be glycogen elongation.

From a structural point of view, AS consists of five domains, named N, A, B, B', and $\mathrm{C}^{8}$ (Fig. 1). Domains A, B, and $\mathrm{C}$ are common to family 13 enzymes, with the characteristic $(\beta / \alpha)_{8}$-barrel catalytic A-domain, whereas the helical $\mathrm{N}$-terminal domain and the $\mathrm{B}^{\prime}$-domain, corresponding to an extended loop 7 of the barrel, are specific to AS. The $3 \mathrm{D}$-structures of AS in complex with sucrose ${ }^{9}$ or maltoheptaose $^{10}$ enabled us to localize the active site as well as

The Supplementary Material referred to in this article can be found at http://www.interscience.wiley.com/jpages/0887-3585/suppmat

*Correspondence to: Magali Remaud-Siméon, Laboratoire de Biotechnologie-Bioprocédés, UMR CNRS 5504, UMR INRA 792, INSA DGBA, 135, avenue de Rangueil, 31077 Toulouse Cedex 04, France. E-mail: remaud@insa-toulouse.fr

Received 20 June 2005; Revised 14 April 2006; Accepted 27 April 2006

Published online 16 October 2006 in Wiley InterScience (www. interscience.wiley.com). DOI: $10.1002 /$ prot.21083 


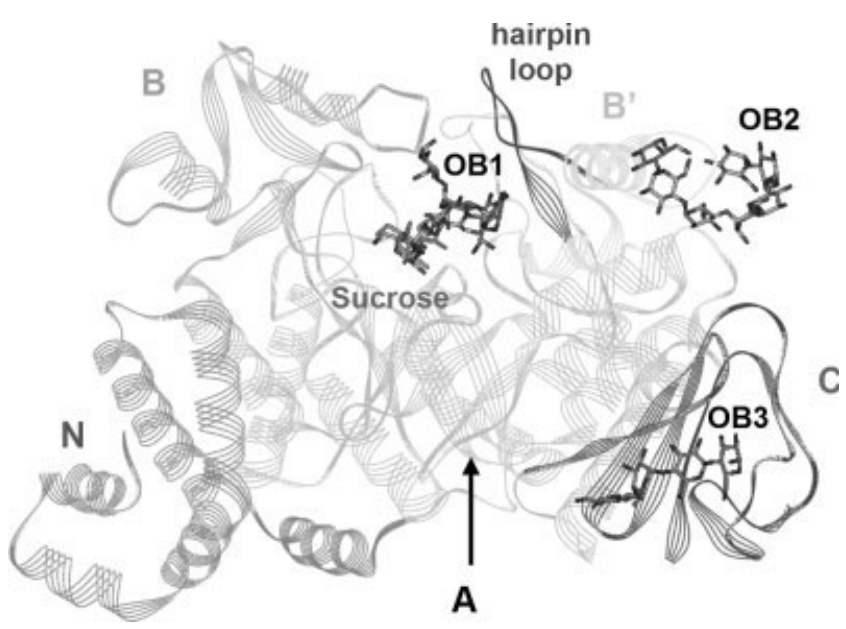

Fig. 1. Overall structure of AS with a sucrose molecule occupying the active site and three maltooligosaccharides bound in the oligosaccharides binding sites OB1 to OB3. The B'-domain hairpin loop Gly 433-Gly449 is shown in dark grey.

three additional oligosaccharide binding sites (OB1-OB3) (Fig. 1). The active site, consisting of -1 and +1 subsites $^{9}$ (according to the nomenclature defined by Davies and Henrissat ${ }^{11}$ ), is situated at the bottom of a narrow pocket delimited by a salt bridge. The first oligosaccharide binding site OB1 spans over the active site and five additional acceptor subsites $(+2$ to +6$){ }^{10}$ The two other sites OB2 and OB3, located at the enzyme surface, are expected to provide additional binding sites for acceptor molecules. ${ }^{10}$ The catalytic active site residues are the nucleophile Asp286, the general acid/base catalyst Glu328, and Asp393 that assists catalysis. ${ }^{8,12}$ The catalysis proceeds via a double-displacement mechanism involving the formation of a covalent glucosyl-enzyme intermediate ${ }^{13-15}$ that has recently been characterized by X-ray crystallography. ${ }^{16}$

The AS-specific B'-domain (residues 395-460) is expected to be involved in the polymerase activity, notably via conformational movements. Indeed, we have recently shown by fluorimetry experiments that the addition of sucrose or glycogen induces enzyme conformational changes, supposed to involve mainly the $\mathrm{B}^{\prime}$-domain. ${ }^{5}$ This corroborates the observations we made on the basis of the comparison of AS 3D-structures, alone or in complex with various substrates. ${ }^{8-10}$ It is also in agreement with the flexibility of a hairpin loop (Gly433-Gly449) belonging to the B'-domain, recently revealed in a robotics modeling study. ${ }^{17}$ Interestingly, the corresponding loop 7 has been shown to be flexible in pig pancreatic $\alpha$-amylase ${ }^{18,19}$, in human salivary $\alpha$-amylase ${ }^{20}$ and also in barley $\alpha$-amylase 2 , where the amplitude of the loop motion has been carefully explored and described using molecular mechanics. ${ }^{21}$

The recent fluorimetry ${ }^{5}$ and robotics ${ }^{17}$ results, combined with AS structural data, ${ }^{8-10}$ suggest a strong involvement of the $\mathrm{B}^{\prime}$-domain in glycogen binding and elongation, possibly associated to structural changes. To further investigate this phenomenon, we have applied a molecular modeling approach to mimic glycogen docking at the enzyme surface. The conformational analysis of the docking solutions, com- bined to robotics calculations and site-directed mutagenesis experiments, provided fruitful information on the AS specificity towards glycogen and enabled us to propose an advanced dynamic mechanism of glycogen elongation.

\section{MATERIALS AND METHODS}

\section{Molecular Modeling}

The molecular modeling was carried out on Silicon Graphics computers with the Accelrys packages (Accelrys, San Diego, CA). Molecular displays and energy minimizations were performed with InsightII, Biopolymer, and Discover modules. For all calculations, the CFF91 force-field with the steepest descent minimization was selected.

\section{Glycogen Docking Definition of the starting coordinates}

$\mathrm{X}$-ray data of the complex between AS (inactive mutant E328Q) and maltoheptaose (G7) fragments bound at the OB1 and OB2 binding sites ${ }^{10}$ were used as starting coordinates for the modeling of glycogen docking (1MW0.pdb). To spare cpu time, the protein was reduced to the residues involved in the complex, which included all $\beta \rightarrow \alpha$ loops belonging to the $(\beta / \alpha)_{8}$-barrel and residues from the neighboring C-terminal ends of $\beta$ strands and $\mathrm{N}$-terminal ends of $\alpha$ helices. This procedure led to an enzyme of 309 residues out of the 628 where the following segments have been included in the computations: 102-112, 131-163, 182-278, 284-304, 328-332, 348-358, 387-464, 484-536. However, the term "enzyme" will be used for this truncated protein in the following text. The 14 glucosyl residues of the two maltoheptaose molecules docked in OB1 and OB2 were kept in their crystallographic positions with the exception of the three residues involved in the branch construction (Fig. 2):

- the two loosely defined glucosyl moieties bound at OB1 subsites +5 and +6 (reducing end);

- the glucosyl unit at the reducing end of G7 occupying OB2 site. The penultimate glucose ring on the reducing side remains fixed as it is tightly maintained by a stacking interaction with aromatic residue Phe417.

\section{Position of the branch}

Among the 14 possible positions of the $\alpha-1,6$ branch between the two maltoheptaose molecules (i.e. from each glucosyl residue), only two possibilities were relevant regarding the structural constraints. These two solutions display a branch that is neither too distant from the active site nor too deeply buried inside the catalytic groove:

1. The $\alpha-1,6$ junction is positioned on the 6 th glucosyl moiety starting from the nonreducing end of the G7 bound in OB2 [Fig. 2(A)]. The degree of polymerization (DP) of the fragment is then in accordance with the average glycogen branch length (DP 11). 
A

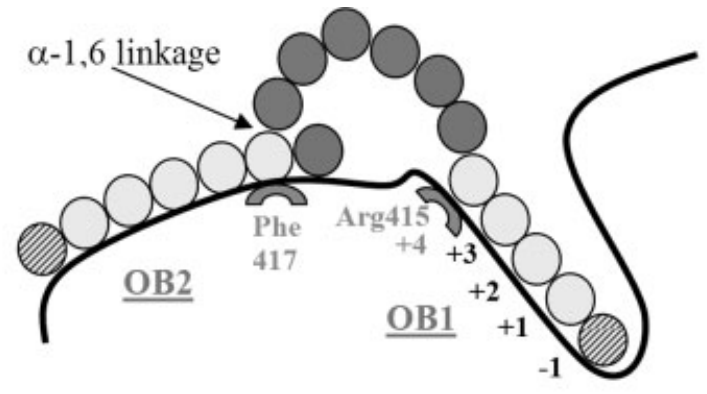

B

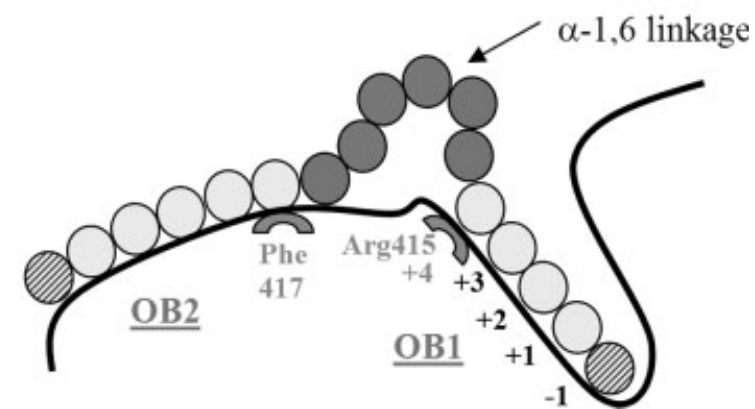

Fig. 2. Schematic representation of the models constructed: (A) with the branch on OB2; and (B) with the branch on OB1. The glucosyl units of the branch added or the crystallographic ones allowed to move are colored in grey. The nonreducing end residues of the chains are striped.

2. The $\alpha-1,6$ junction is positioned on the 7 th glucosyl ring starting from the non-reducing end of the G7 docked in OB1 [Fig. 2(B)].

\section{Construction and energy minimization of the models}

The branch is constructed by the addition of glucosyl residues, first through an $\alpha-1,6$ linkage carried by one of the two G7 molcules bound in OB1 or OB2, then through $\alpha-1,4$ linkages to join the second G7. It is known from the literature that an isomaltose moiety has six low energy conformations, ${ }^{22}$ whereas a maltose moiety displays only four. ${ }^{23}$ Consequently, the construction of the $\alpha-1,6$ branch point necessitates to explore the six isomaltose conformations in the corresponding $(\varphi, \psi)$ space. Similarly, for any glucose ring added via an $\alpha-1,4$ linkage, the four maltose conformations were tested to locate the minima in the corresponding $(\varphi, \psi)$ map. To reduce the number of solutions (in theory $6 \times 4^{n-1}$ for a branch with $\mathrm{n}$ residues added), geometrical criteria were considered. So, this iterative construction allowed a correct orientation of the branch towards the G7 in order to be joined, a relevant docking of a branched fragment via as many interactions as possible with the enzyme (except for $\mathbf{I}^{\prime}$-model) and also avoided volume collision. This strategy led to the construction and energy minimization of 13 branches. In the first calculations, the backbone of all the enzyme residues not directly in contact with the substrate fragments was kept fixed as well as the crystallographic glucosyl residues (except the three residues described in Initial substrate). The constraints were kept for 5000 iterations, then relaxed for 1000. Afterwards, potential energies of both enzyme and substrate were calculated as well as their energy of interaction. Substrate and docking energies were expressed relative to the lowest energy which was normalized to zero.

\section{Robotics of $\mathbf{B}^{\prime}$-Domain Hairpin Loop Gly433-Gly449}

Among the internal motions that can be simulated by molecular dynamics calculations, loop motions are rather high energy motions, hard to sample in a period of time shorter than a nanosecond. Moreover such a long dynamics simulation would neither guarantee a complete description of the loop motion nor the evaluation of its amplitude. Here, an alternative strategy, called "robotics" and recently used with success to describe barley $\alpha$-amylase loop 7 motion, ${ }^{21}$ has been developed.

\section{Robotics method}

Since the hairpin loop of the B'-domain is bordered by two glycine residues (Gly433 and Gly449), the movement of loop 7 is likely to be a flap and back over the active site, as it was recently proposed by Cortes et al. ${ }^{17}$ To model the trajectory of this loop, the movement is decomposed into tiny steps supposed to be a relevant description of the reaction path. Using this procedure, we have partitioned the system into rigid and mobile segments, defined by two barycenters:

i. $\mathbf{X}$ representing the rigid segment. It corresponds to the truncated enzyme (already described in Glycogen docking) without including the $\mathrm{B}^{\prime}$-domain, and is defined by the center of mass between the catalytic residues Asp286, Glu328, and Asp393.

ii. $\mathbf{X}^{\prime}$ representing the mobile hairpin loop of $\mathrm{B}^{\prime}$-domain. It is defined by the backbone chain of residues Gly433Gly449.

To give a continuous description of the movement, the calculations comprised successive $0.2 \AA$ displacements of $\mathbf{X}^{\prime}$ towards $\mathbf{X}$ and the reverse movement of $\mathbf{X}^{\prime}$ back from $\mathbf{X}$. Starting from crystallographic coordinates (described below), the distance constraints encompassed the decrease and increase of distance between $\mathbf{X}$ and $\mathbf{X}^{\prime}$.

\section{Definition of the starting coordinates}

Two trajectories of the domain motion have been calculated:

i. One with a sucrose molecule occupying the active site, for which the crystallographic data of E328Q: sucrose complex ${ }^{9}$ were used as starting coordinates. This stage corresponds to the initial step of the catalysis, before sucrose cleavage.

ii. The other one with a glycogen fragment bound at the OB1 acceptor site, in a position permitting a new sucrose molecule to enter the active site. This corresponds to a later step of the catalytic cycle occurring after fructose departure, glucosyl transfer, and repositioning of glycogen fragment. The starting model chosen was model I 
that was obtained from the docking study described above, and where -1 and +1 subsites were emptied. However, the displacement inducing the cleft closure being not sterically feasible, only the backward motion of $\mathbf{X}^{\prime}$ from $\mathbf{X}$ was explored.

In the presence of either sucrose or the model $\mathbf{I}$ branched ligand, the starting structures have a similar $\mathrm{X}-\mathrm{X}^{\prime}$ distance of $19.3 \AA$. In both cases, the enzyme corresponds to the truncated form defined in Glycogen docking.

\section{Energy minimization}

For each $0.2 \AA$ displacement step computed by the robotics, 2000 minimization iterations were performed with the backbone of AS residues kept fixed, except for $\mathrm{B}^{\prime}$ domain amino acids that are free to move, and without any constraints on the ligand. The calculation with constraints was then followed by a relaxation with 500 minimization iterations to let the system adapt to its new position. After each distance restraints and coordinates relaxation, the distance $\mathbf{X}-\mathbf{X}^{\prime}$ was checked to ensure it had stayed within the defined distance range and energies of the complex as well as docking energies were calculated. At the end of the procedure, the series of $\mathbf{X}-\mathbf{X}^{\prime}$ displacements was merged into a final trajectory. This finally led to a loop motion oscillating from a closed to an open conformation.

\section{Plasmid and Bacterial Strains}

The pGST-AS encoding glutathione S-transferase (GST, $26 \mathrm{kDa})$ fused to amylosucrase $(\mathrm{AS}, 70 \mathrm{kDa})^{2}$ was used to express the fusion gene and for site-directed mutagenesis. E. coli strain JM109 was used as the host of pGST-AS encoding wild-type or mutated AS.

\section{Site-Directed Mutagenesis}

Site-directed mutagenesis of the as gene was carried out with the QuikChange ${ }^{\mathrm{TM}}$ site-directed mutagenesis kit (Stratagene), as previously described. ${ }^{12}$ The procedure utilized the pGST-AS double-stranded DNA vector and two synthetic oligonucleotide primers each complementary to opposite strands of the vector. Primers contained the desired mutation (bold in the following sequences) and were designed to create a restriction site that was used to screen the correct mutation. The following primers were used to construct the F417A mutant:

F417A_for: 5'-GGCTACGACCACCGCCAAGCCCTCAACCGGTTCTTCGTCAACCG AgeI

F417A_rev: 5'-CGGTTGACGAAGAACCGGTTGAGGGCTTGGCGGTGGTCGTAGCC AgeI

The mutations were confirmed by DNA sequencing (Genome Express, Grenoble, France).

\section{Enzyme Preparation}

$E$. coli carrying the recombinant pGST-AS plasmid encoding the wild-type and mutated as gene was grown on LB (Luria-Bertani) medium containing ampicillin $(100 \mu \mathrm{g} /$
$\mathrm{mL}$ ) and isopropyl- $\beta$-thiogalactopyranoside $(1 \mathrm{mM})$ for $10 \mathrm{~h}$ at $30^{\circ} \mathrm{C}$. The cells were harvested by centrifugation $(8000 \mathrm{~g}$, $10 \mathrm{~min}, 4^{\circ} \mathrm{C}$ ), resuspended, and concentrated to an $\mathrm{OD}_{600 \mathrm{~nm}}$ of 80 in PBS buffer (140 mM NaCl, $2.7 \mathrm{~m} M \mathrm{KCl}, 10 \mathrm{~m} M$ $\mathrm{Na}_{2} \mathrm{HPO}_{4}$, and $1.8 \mathrm{mM} \mathrm{KH} \mathrm{PO}_{4}, \mathrm{pH}$ 7.3). The intracellular enzyme was extracted by sonication and $1 \%(\mathrm{v} / \mathrm{v})$ Triton $\mathrm{X}-100$ was added to the extract and mixed for $30 \mathrm{~min}$ at $4^{\circ} \mathrm{C}$. After centrifugation $\left(10,000 \mathrm{~g}, 10 \mathrm{~min}, 4^{\circ} \mathrm{C}\right)$, the supernatant was used as the crude extract for enzyme assay.

\section{Enzyme Assays}

The AS assays were carried out at $30^{\circ} \mathrm{C}$ in $50 \mathrm{mM}$ Tris$\mathrm{HCl} \mathrm{pH} 7.0$ in the presence of $146 \mathrm{mM}$ sucrose alone or supplemented with $30 \mathrm{~g} / \mathrm{L}$ glycogen, using crude supernatant as the enzyme source (wild-type AS or F417A mutant), with appropriate dilution calculated to approach the same activity for both reactions. The activity of the GST-AS fusion protein being equivalent to that of AS (data not shown), the GST tag was not removed for the assays. The activities were determined by measuring the initial rates of formation of reducing sugars (fructose and glucose) released from sucrose per minute and per liter of crude supernatant. The activity of the F417A variant was expressed relative to the activity of the wild-type AS, considering the expression level of both enzymes estimated by SDS-PAGE electrophoresis.

\section{Carbohydrate Analysis}

The concentration of reducing sugars was measured by the dinitro-salicylic acid method, using fructose as a standard. ${ }^{24}$

The soluble products (glucose, sucrose isomers, and maltooligosaccharides) synthesized by wild-type AS and the F417A mutant after $20 \mathrm{~h}$ reaction were identified by High Performance Anion Exchange Chromatography (HPAEC) using a Dionex Carbo-Pack PA100 column at $25^{\circ} \mathrm{C}$. A mobile phase $(150 \mathrm{~m} M \mathrm{NaOH})$ was used at $1 \mathrm{~mL} / \mathrm{min}$ flow rate with a sodium acetate gradient (from 6 to $600 \mathrm{mM}$ in $60 \mathrm{~min}$ ). Detection was performed using a Dionex ED40 module with a gold working electrode and an $\mathrm{Ag} / \mathrm{AgCl} \mathrm{pH}$ reference. No quantification of the products formed was carried out.

\section{RESULTS AND DISCUSSION}

\section{Construction of Branching Models}

In the E328Q:maltoheptaose complex, ${ }^{10}$ two maltoheptaose molecules are bound in OB1 and OB2 sites with their reducing ends facing each other. Our strategy was to join these two maltoheptaose molecules via an $\alpha-1,6$ branch in order to mimic a glycogen or amylopectin fragment. From the two branching points chosen (see Material and Methods and Fig. 2), 13 glycogen fragment models were constructed. These models showed a correct orientation towards the second G7 to be joined, as well as a good proximity of the branch to the enzyme surface (except in model $\mathbf{I}^{\prime}$, corresponding to an elongated branch) and had no steric hindrance or any van der Waals volume collision. Among these 13 models, four correspond to a branching on G7 bound 
TABLE I. Characteristics of the Four Glycogen Fragment Models Selected From Geometrical and Energetic Criteria

\begin{tabular}{lccccc}
\hline Model & Branching position $^{\mathrm{a}}$ & $\begin{array}{c}\text { Branch } \\
\text { size }^{\mathrm{b}}\end{array}$ & $\begin{array}{c}\text { Protein energy } \\
(\mathrm{kcal} / \mathrm{mol})\end{array}$ & $\begin{array}{c}\text { Relative substrate } \\
\text { energy (kcal/mol) }\end{array}$ & $\begin{array}{r}\text { Relative docking } \\
\text { energy }(\mathrm{kcal} / \mathrm{mol})\end{array}$ \\
\hline I & 6th glucose OB2 & DP 11 & -7080 & 27 & 34 \\
I $^{\prime}$ & 6th glucose OB2 & DP 15 & -7083 & 60 & 0 \\
II & 6th glucose OB2 & DP 12 & -7082 & 35 & 33 \\
III & 7th glucose OB1 & DP 10 & -7085 & 0 & 28 \\
\hline
\end{tabular}

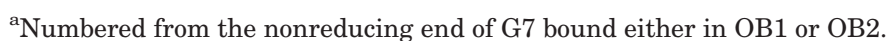

${ }^{\mathrm{b}} \mathrm{DP}$, degree of polymerization.

in OB1 and nine on OB2. After energy minimizations, only four models, numbered I, I', II, and III, were acceptable from geometrical and energetic points of view (Table I, Fig. 3). In models I, I', and II, the branch is linked to the maltoheptaose docked in OB2. In model III, the branch is connected to the maltopheptaose docked in OB1. The existence of various modes of docking with quite similar energies indicates that AS accepts several different ligand binding modes. Overall, this reflects the multiple positions that glycogen can adopt onto AS.

\section{Characteristics of the Four Models}

Models I, II, and III were constructed to "bind" as strongly as possible to the enzyme surface (Fig. 3I, 3II, and 3III). The length of the oligomers was restricted to a value between 10 and 12 and only $3-5$ residues were added to the glucosyl residues found in the structure. In contrast, model $\mathbf{I}^{\prime}$, derived from model $\mathbf{I}$, is longer (DP 15) and located farther from the enzyme surface (Fig. 3I'). This model represents an intermediate elongation product, coming from the initial docking mode of I-model. Interestingly, this elongated chain adopts the helical conformation of a native amylose chain with its glycosidic linkages describing repeatedly $(\phi, \psi)$ dihedral angles $(\varphi, \psi=$ $\mathrm{O}^{\prime}$-C1'-O4-C4, C1'-O4-C4-C5) close to the characteristic $\left(86^{\circ},-146^{\circ}\right)$ values. All $\alpha-1,4$ glucosidic linkages built to connect the second maltoheptaose are in stable conformations. ${ }^{23}$ However, it has been necessary to position one glucosidic linkage in the $E$ conformation $\left(\varphi, \psi ; 85^{\circ}, 84^{\circ}\right)$. This conformation is separated from the contiguous regions $\mathrm{A}$ $\left(56^{\circ},-151^{\circ}\right), \mathrm{B}\left(114^{\circ},-133^{\circ}\right)$, and $\mathrm{C}\left(133^{\circ},-108^{\circ}\right)$ of the potential energy contour map of $\alpha-1,4$ disaccharide. However, no glucosyl ring distortion is observed after energy minimization. The potential energies of the protein are very similar for the four models and the relative substrate energies increase smoothly with the substrate size, indicating that neither deformations nor artifacts were induced during calculations (Table I). Surprisingly, the relative docking energies clearly show that model $\mathbf{I}^{\prime}$ displays the best enzyme-substrate interaction, suggesting that some unfavorable surface interactions are present in the other three models. However, the addition of the relative substrate and docking energies shows that model III is the most favorable from an energetic point of view.

The four enzyme:substrate complexes show a similar hydrogen bonding network and van der Waals interactions pattern. Residues Asp401, Asp413, Arg415, Arg420,
Asp427, Asp444, Tyr412, and Phe417 participate in the binding, either through hydrogen bonds or stacking interactions. In addition, the hairpin loop formed by residues Gly433-Gly449 (pink in Fig. 3) provides a structural support to the branching saccharide moiety. This loop seems to be stabilized by the helix Ser410-Phe422 (orange in Fig. 3) that is a key structural feature of the $\mathrm{B}^{\prime}$-domain (Asp394-Asp460). Markedly, among the residues that participate to the binding of the monosaccharide that was added for the branch construction, none provides strong stacking interactions. For instance, residue Tyr412 (Fig. 3), which occupies a key position between OB1 and OB2, is never in the optimal conformation to give a strong stacking interaction. In agreement, replacement of this tyrosine by a glycine had no effect on enzyme activity (data not shown). Consequently, the only stacking patch is offered by Phe417, a residue that has already been described as providing the main binding strength of the OB2 site. ${ }^{10}$

\section{Role of Phe417 and Arg415 in Glycogen Binding}

To confirm the role of Phe417, we have suppressed its possible stacking interaction by changing it to an alanine. Measurement of the initial release of reducing sugars (fructose and glucose) revealed that the activity of the F417A mutant in the presence of $146 \mathrm{mM}$ sucrose and $30 \mathrm{~g} / \mathrm{L}$ glycogen corresponds to $29 \%$ of the wild-type AS activity. Besides, glycogen induces only a 50-fold activity increase for F417A, in comparison with the 98-fold activation observed for the wild-type enzyme in similar conditions. The superimposition of the HPAEC chromatography profiles of the soluble products, obtained with wildtype AS and F417A mutant, shows that turanose and maltooligosaccharides are present only in the F417A assay profile (Fig. 4). The F417A profile also shows that sucrose has not been totally consumed because of the reduced activity of F417A mutant. This demonstrates that the F417A mutant (unlike wild-type AS) still synthesizes soluble oligosaccharides (sucrose isomers, maltooligosaccharides) in the presence of glycogen to the detriment of glycogen elongation. These results demonstrate that Phe417 exposed at AS surface provides an easily accessible docking platform for a glycogen fragment. Another residue, Arg415, situated at the entrance of the channel to the active site (subsite +4 of OB1 site), contributes strongly to the affinity of AS for glycogen. It was shown that the replacement of Arg415 by an alanine significantly reduces the affinity of AS towards glycogen, 

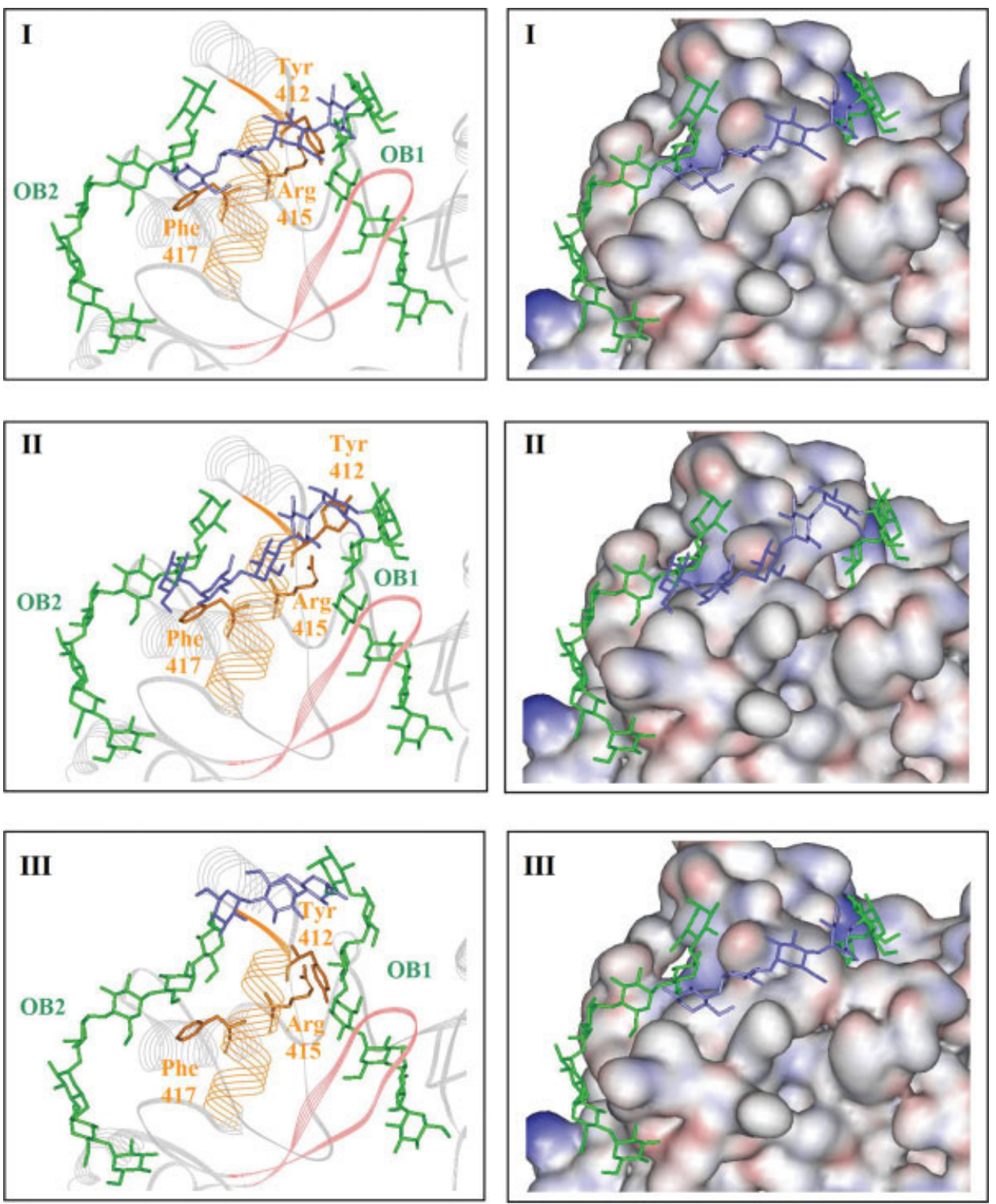

Fig. 3. Representation of the four models (I, II, III, and I') selected. The crystallographic glucosyl residues are colored in green and the glucosyl units added are in blue. The helix 410-422 is colored in orange and the hairpin loop $433-449$ is in pink.

whose activating effect on catalysis decreased by 12 -fold. ${ }^{5}$ Then, it can be suggested that, once glycogen is anchored onto AS, the branches could be subsequently directed towards the site OB1 via an interaction with Arg415. Interestingly, Phe417 and Arg415 belong to the helix Ser410-Phe422 of the B'-domain, which occupies a central position between OB1 and OB2 sites and provides a structural fundament for the hairpin loop Gly433-Gly449.

\section{Flexibility of $\mathbf{B}^{\prime}$-Domain Hairpin Loop \\ Gly433-Gly449 \\ Trajectory with sucrose}

The trajectory of the hairpin loop Gly433-Gly449 has been first calculated with a sucrose molecule occupying the active site. The energy diagram of E328Q:sucrose complex was plotted versus $\left[\mathrm{X}-\mathrm{X}^{\prime}\right]$ distance (Fig. 5). Interestingly, the diagram describes a symmetric curve with an energy decrease and increase apart from the medium crystallographic position (XR). It should be noted that in the crystal structure, the tip of the loop (residues 441 and 442) is involved in crystal packing with the N-terminal of a neighboring molecule that could be responsible for the local energy maximum observed. However, the potential energy value of the complex at the $\mathrm{XR}$ position is an experimental value that can serve as an energy threshold reference. Consequently, the conformations with potential energy beyond this value were discarded. Actually, all the positions for which the potential energy rises steeply after the threshold value were shown to arise from loop conforma- 

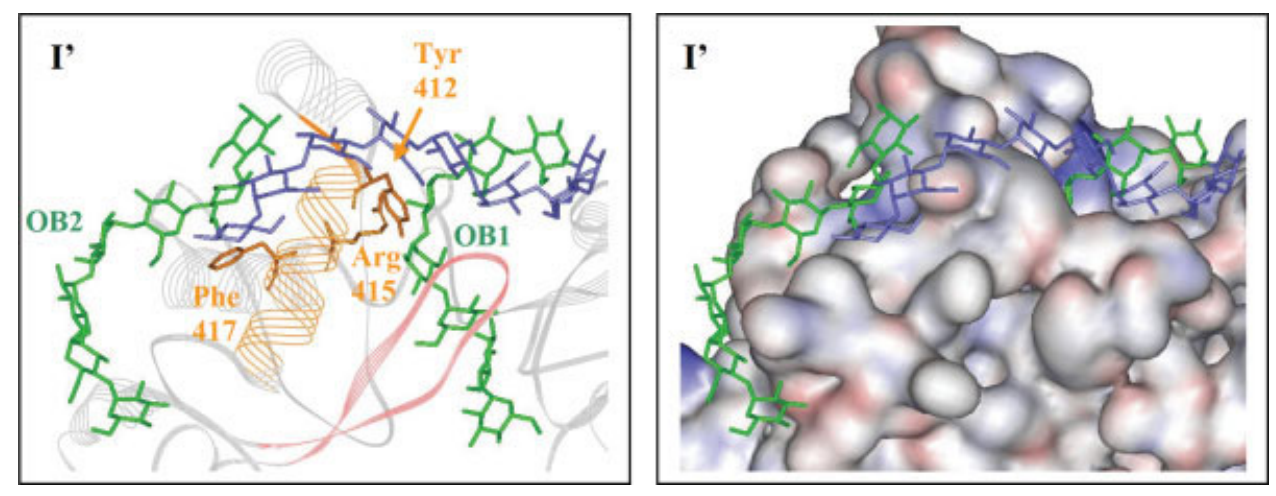

Figure 3. (Continued).

tions inducing steric hindrance with the other part of the enzyme. At this threshold, three positions of the hairpin loop were selected: the crystallographic one as the XR medium position and its isoenergetic counterparts on the diagram. These latter experimental threshold values were used to border out a back and forth motion of $13 \AA$ of amplitude for the hairpin loop. The B'-domain helix Ser410Phe422 stabilizes the hairpin conformation that is not distorted during all movement. The two isoenergetic loop conformations are located at -5 and $+8 \AA$ away from XR, defining a "closed" and an "open" position for the hairpin loop (Fig. 5) [Supplementary material]. In addition to these three positions, two minima were found, suggesting two highly probable positions. When the loop reaches the closed position, it markedly reduces the volume of the cleft, thus destabilizing the fructose moiety of the sucrose. This $5 \AA$ backward motion could favor the release of the leaving group fructose once sucrose is cleaved. Conversely, the complete opening of the hairpin loop induces a tremendous enlargement of the cleft that may favor the entrance of a second sucrose molecule. This motion could facilitate the supply of glucose to the OB1 donor site before the transfer step. Such a back and forth motion would move the catalytic residue Asp393 bringing it closer to its catalytic counterparts Asp286 and Glu328 for the linkage cleavage and then removing it from them again to favor the release of the leaving group. These results confirm the importance of the hairpin loop Gly433-Gly449 already suggested by Cortes et al. ${ }^{17}$ and attest that $\mathrm{B}^{\prime}$-domain motion is mainly driven by this specific hairpin loop. In addition, this molecular modeling study corroborates nicely our previous fluorimetry experiments that revealed the existence of conformational changes induced through sucrose binding on $\mathrm{AS}^{5}$ Finally, it should be noted that the two glycine residues that act as hinge residues are highly conserved among putative AS of known sequence (data not shown), suggesting a similar flexibility for the $\mathrm{B}^{\prime}$-domain of these enzymes.

\section{Trajectory with model I}

To investigate the influence of the hairpin loop Gly433Gly449 flexibility onto glycogen elongation, a similar robotic study has been performed with model I where -1 and +1 subsites were emptied. This corresponds to an

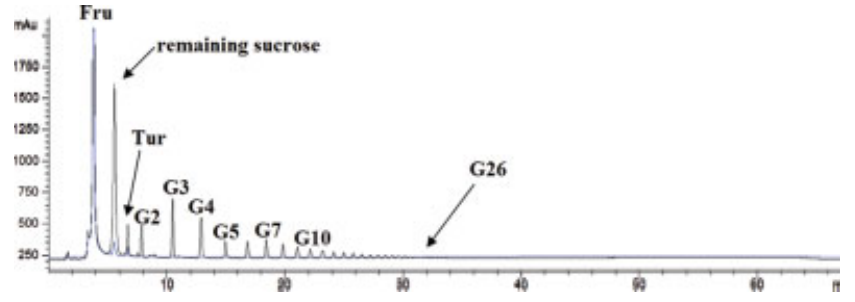

Fig. 4. Superimposition of the Dionex High Performance Anion Exchange Chromatography profiles of the soluble fractions obtained after $20 \mathrm{~h}$ reaction, using wild-type AS (in blue) or F417A variant (in black) in the presence of $146 \mathrm{mM}$ sucrose and $30 \mathrm{~g} / \mathrm{L}$ glycogen. Fru: fructose, Tur: turanose, $\mathrm{G}_{n}$ : maltooligosaccharides of DP of $n$.

advanced step of the catalytic cycle, after fructose departure, glucosyl transfer, and repositioning of glycogen fragment to permit a new sucrose moecule to enter the active site. Because of steric hindrance between substrate and enzyme, the trajectory computed is half the previous one and includes the range from the XR position to the position already identified as an energy minimum conformation in the open state (Fig. 5). During its $4 \AA$ movement, the hairpin loop motion was characterized by a significant change in the positions of the hairpin edge (Phe436Asp444) and the residues Gly433 and Gly449. Here, we paid special attention to the interactions between the enzyme and the glycogen fragment during the opening of the hairpin loop. The docking energy diagram of the trajectory is shown in Figure 6. Interestingly, the docking energy between the protein and the substrate first decreased regularly from -327 to $-343 \mathrm{kcal} / \mathrm{mol}$ when the $\mathrm{X}-\mathrm{X}^{\prime}$ distance increased from 19.3 to $21.2 \AA$, strengthening glycogen anchoring as the loop opens. Passed that position, the docking energy increased, suggesting a weakening in the binding. During the simulations, neither distortion from ${ }^{4} \mathrm{C}_{1}$ chair conformations for the substrate nor dihedrals out of the $(\varphi, \psi)$ allowed regions were observed for the glycosidic linkages. Interestingly, the ligand part docked in OB1 and, particularly the glucose moiety located at subsite +2 , tended to slip away from its initial position to reach subsite +3 . Similarly, the glucose at +3 tended to shift towards +4 and so on. This occurs without passing over high energy barriers. Thus, besides favoring sucrose supply, the hairpin loop movement could 

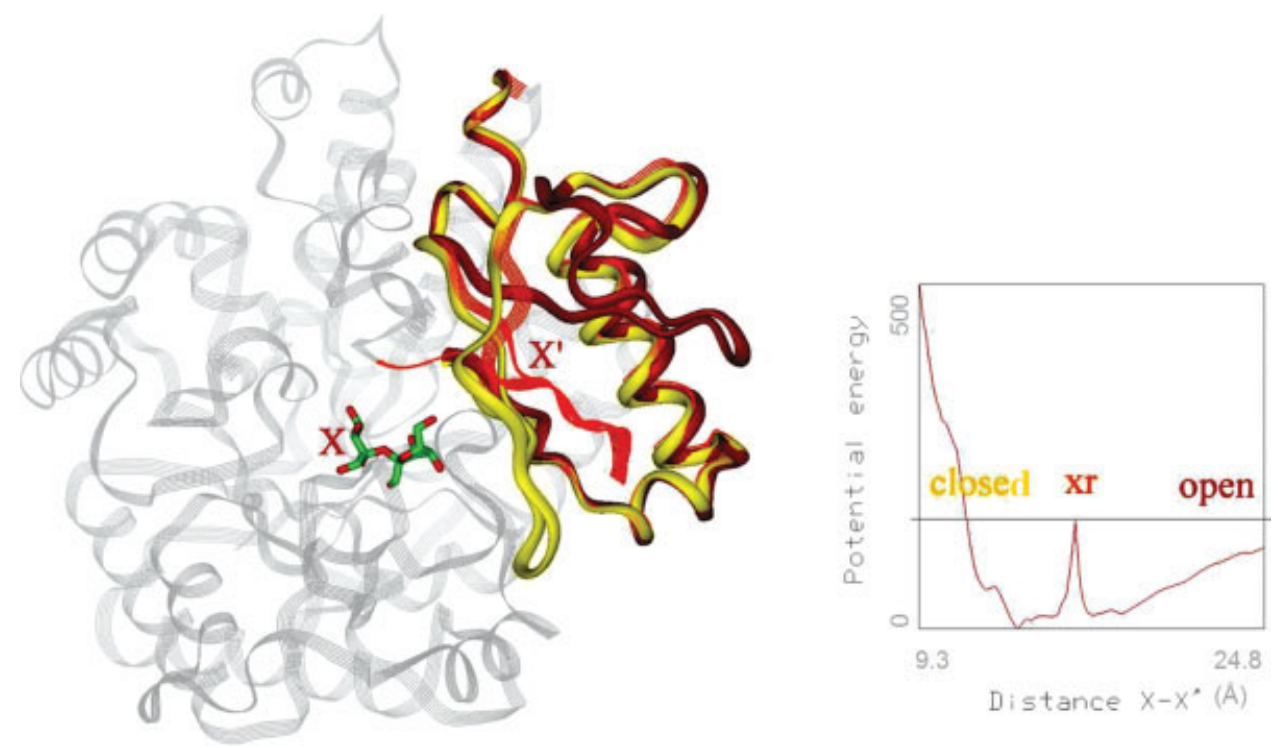

Fig. 5. Robotics trajectory of $\mathrm{B}^{\prime}$-domain with sucrose (green) bound in the active site: closed (yellow), crystallographic (xr) (orange), and open positions (red) of the hairpin loop Gly433-Gly449. The corresponding relative complex potential energy versus distance is plotted.
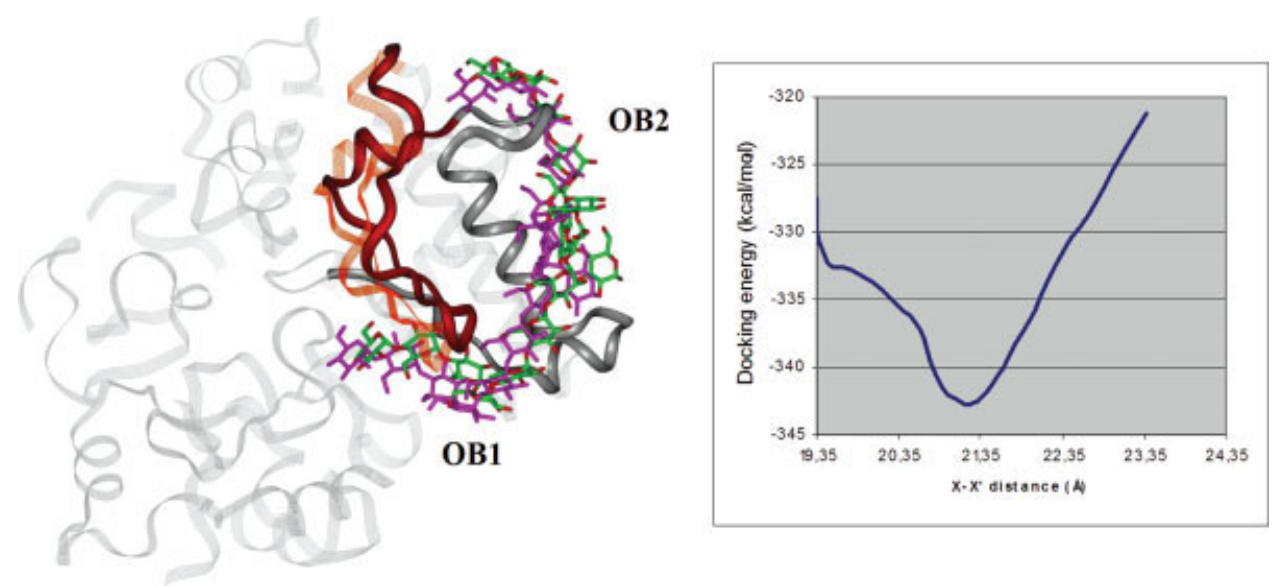

Fig. 6. Robotics trajectory of $\mathrm{B}^{\prime}$-domain with the model I glycogen fragment, cristallographic (orange) and substantially open position (red) of the hairpin loop Gly433-Gly449. The corresponding model I glycogen fragments are colored in pink and by atom type, in the xr and open positions of the hairpin-loop, respectively. The corresponding docking energy versus distance is plotted.

assist the in-coming and out-going of acceptor molecules to the active site, favoring the positioning of the acceptor molecule, hence influencing the transglucosylation step and consequently the polymerization reaction. Moreover, this loop motion could significantly decrease the energy penalty during these various steps.

It is interesting to note that, all along the path, the ligand part fixed in OB2 stayed bound to the enzyme, notably via the key $\mathrm{B}^{\prime}$-domain residue Phe417. We propose that glycogen is first captured on the surface of AS, then guided, notably via Arg415, towards OB1 with its nonreducing end occupying the subsite +1 for glucosylation. The OB2 surface binding site is undoubtedly strongly involved in the capture of glycogen. OB3 could play the same role and provide additional anchoring sites, contrib- uting to the remarkable affinity of AS towards glycogen. Mutations directed towards OB3 residues need now to be attempted to confirm this hypothesis.

\section{CONCLUSIONS}

Our study provides for the first time new insights into both the structural determinants involved in glycogen docking and the dynamics of glycogen elongation. A "semiprocessive" mechanism in which a glycogen branch remains captured at the OB2 surface site of the enzyme while another one is elongated can be proposed. The branch elongation occurs through acceptor in-coming and out-going movements assisted by a back and forth motion of the hairpin loop Gly433-Gly449. After each transglucosylation re- 
action, the elongated chain must move out of the active site groove (at least by two subsites in the positive direction), to empty subsites -1 and +1 and to enable a new sucrose molecule to reach the active site. Elongation is expected to continue until the branch reaches a critical size changing the thermodynamic balance and causing the release of modified glycogen. This latter can again be anchored on AS surface for a new branch elongation. Such a binding mode of glycogen acceptor that always remains in close proximity of the active site results in water exclusion, which is in agreement with the absence of hydrolysis observed in the presence of glycogen. ${ }^{6}$

To conclude, the high specificity of AS for glycogen results from a strong anchoring of the glucan at the surface of the enzyme providing an efficient guidance of the nonreducing ends of the branches towards the active site.

\section{ACKNOWLEDGMENT}

The authors thank Dr. Isabelle André for reading the manuscript.

\section{REFERENCES}

1. Hehre EJ, Hamilton DM, Carlson AS. Synthesis of a polysaccharide of the starch-glycogen class from sucrose by a cell-free bacterial enzyme system (amylosucrase). J Biol Chem 1949;177:267279.

2. Potocki de Montalk G, Remaud-Simeon M, Willemot RM, Planchot V, Monsan P. Sequence analysis of the gene encoding amylosucrase from Neisseria polysaccharea and characterization of the recombinant enzyme. J Bacteriol 1999;181:375-381.

3. Coutinho PM, Henrissat B.Carbohydrate-active enzymes. Available at http://afmb.cnrs-mrs.fr/CAZY/

4. Potocki de Montalk G, Remaud-Simeon M, Willemot RM, Sarçabal P, Planchot V, Monsan P. Amylosucrase from Neisseria polysaccharea: novel catalytic properties. FEBS Lett 2000;471: 219-223.

5. Albenne C, Skov LK, Mirza O, Gajhede M, Feller M, D'Amico S, André G, Potocki-Véronèse G, van der Veen BA, Monsan P, Remaud-Simeon M. Molecular basis of the amylose-like polymer formation catalysed by Neisseria polysaccharea amylosucrase. J Biol Chem 2004;279:726-734.

6. Potocki de Montalk G, Remaud-Simeon M, Willemot RM, Monsan P. Characterisation of the activator effect of glycogen on amylosucrase from Neisseria polysaccharea. FEMS Microbiol Lett 2000;186:103-108.

7. Sabaté-Rolland A, Planchot V, Véronèse G, Monsan P, Colonna P. Elongation and insolubilization of $\alpha$-glucans by the action of Neisseria polysaccharea amylosucrase. J Cer Sci 2004;40:17-30.
8. Skov LK, Mirza O, Henriksen A, Potocki de Montalk G, RemaudSimeon M, Sarçabal P, Willemot RM, Monsan P, Gajhede M. Amylosucrase, a glucan-synthesizing enzyme from the $\alpha$-amylase family. J Biol Chem 2001;276:25273-25278.

9. Mirza O, Skov LK, Remaud-Simeon M, Potocki de Montalk G, Albenne C, Monsan P, Gajhede M. Crystal structure of amylosucrase from Neisseria polysaccharea in complex with D-glucose and the active site mutant Glu328Gln in complex with the natural substrate sucrose. Biochemistry 2001;40:9032-9039.

10. Skov LK, Mirza O, Sprogøe D, Dar I, Remaud-Simeon M, Albenne C, Monsan P, Gajhede M. Oligosaccharide ans sucrose complexes of amylosucrase. Structural implications for the polymerase activity. J Biol Chem 2002;277:47741-47747.

11. Davies G, Henrissat B. Structures and mechanisms of glycosyl hydrolases. Structure 1995;3:853-859.

12. Sarçabal P, Remaud-Simeon M, Willemot RM, Potocki de Montalk G, Svensson B, Monsan P. Identification of key amino acid residues in Neisseria polysaccharea amylosucrase. FEBS Lett 2000; 474:33-37.

13. Koshland DE. Stereochemistry and the mechanism of enzymatic reactions. Biol Rev Camb Philos Soc 1953;28:416-436.

14. Svensson B. Protein engineering in the $\alpha$-amylase family: catalytic mechanism, substrate specificity, and stability. Plant Mol Biol. 1994;25:141-157.

15. Uitdehaag JCM, Mosi R, Kalk KH, van der Veen BA, Dijkhuizen L, Withers SG, Dijkstra BW. X-ray structures along the reaction pathway of cyclodextrin glycosyltransferase elucidate catalysis in the $\alpha$-amylase family. Nat Struc Biol 1999;6:5:432-436.

16. Jensen MH, Mirza O, Albenne C, Remaud-Simeon M, Monsan P, Gajhede M, Skov LK. Crystal structure of the covalent intermediate of amylosucrase from Neisseria polysaccharea. Biochemistry 2004;43:3104-3110.

17. Cortes J, Siméon T, Remaud-Siméon M, Tran V. Geometric algorithms for the conformational analysis of long protein loops. J Comput Chem 2004;25:956-967.

18. Qian M, Haser R, Payan F. Structure and molecular model refinement of pig pancreatic $\alpha$-amylase at 2.1 A resolution. J Mol Biol 1993;231:785-799.

19. Gilles C, Astier JP, Marchis-Mouren G, Cambillau C, Payan F. Crystal structure of pig pancreatic $\alpha$-amylase isoenzyme II, in complex with the carbohydrate inhibitor acarbose. Eur J Biochem 1996;238:561-569.

20. Ramasubbu N, Ragunath C, Mishra PJ. Probing the role of a mobile loop in substrate binding and enzyme activity of human salivary amylase. J Mol Biol 2003;325:1061-1076.

21. André G, Tran V. Putative implication of $\alpha$-amylase loop 7 in the mechanism of substrate binding and reaction product release. Biopolymers 2004;75:95-108.

22. Dowd MK, Reilly PJ, French AD. Relaxed-residue conformational mapping of the three linkage bonds of isomaltose and gentibiose with MM3. Biopolymers 1994;34:625-638.

23. André G, Buléon A, Vallee F, Juy M, Haser R, Tran V. Amylose chain behavior in an interacting context. I. Influence of a non-chair ring on the maltose conformations. Biopolymers 1996;39:737-751.

24. Sumner J, Howell S. A method for determination of invertase activity. J Biol Chem 1935;108:51-54. 